\title{
The trace left by signature-change-induced compactification *
}

\author{
Franz Embacher \\ Institut für Theoretische Physik \\ Universität Wien \\ Boltzmanngasse 5 \\ A-1090 Wien \\ E-mail: fe@pap.univie.ac.at \\ UWThPh-1994-55 \\ gr-qc/9411028
}

\begin{abstract}
Recently, it has been shown that an infinite succession of classical signature changes ("signature oscillations") can compactify and stabilize internal dimensions, and simultaneously leads, after a coarse graining type of average procedure, to an effective ("physical") space-time geometry displaying the usual Lorentzian metric signature. Here, we consider a minimally coupled scalar field on such an oscillating background and study its effective dynamics. It turns out that the resulting field equation in four dimensions contains a coupling to some non-metric structure, the imprint of the "microscopic" signature oscillations on the effective properties of matter. In a multidimensional FRW model, this structure is identical to a massive scalar field evolving in its homogeneous mode.
\end{abstract}

*Work supported by the Austrian Academy of Sciences in the framework of the "Austrian Programme for Advanced Research and Technology". 


\section{Introduction}

There are various schemes according to which some of the space-time dimensions compactify to unobservably small scales, while the remaining ones evolve towards cosmologically reasonable sizes. There are (although less) schemes in which the compactification of internal dimensions occurs in a stable way, i.e. robust against small perturbations (see e.g. Refs. [1]-27]). Recently, one additional possibility to achieve this has been added to the list [28]. It relies on the speculative possibility that the metric may change its signature from Euclidean to Lorentzian type (and vice versa) in a classical process [29]-[36]. Each such process happens on a hypersurface whose intrinsic geometries and extrinsic curvatures (the latter describing the embedding into the full manifold) coincide when computed from either side. Moreover, the hypersurface is assumed to be spacelike with respect to the Lorentzian side. The classical signature change models should not be confused with the mixed signature geometries appearing in quantum cosmology [37] 39], in which case the corresponding hypersurfaces are required to have vanishing extrinsic curvature.

In Ref. 28] (and in parts in Ref. [40]), this idea has been applied to a FriedmannRobertson-Walker (FRW) model with spatial sections $\mathbf{S}^{3} \times \mathbf{S}^{6}$ and scale factors $a_{1}$ and $a_{2}$, matter just being represented by a cosmological constant $\Lambda$. It turned out that there are solutions $\left(a_{1}(\tau), a_{2}(\tau)\right)$ such that the metric signature (i.e. the signature of $\left.g_{00}(\tau)\right)$ oscillates rapidly. At large values of the "time" coordinate $\tau$, the observable scale factor $a_{1}$ grows linearly in $\tau$, while $a_{2}$ approaches the scale set by $\Lambda^{-1 / 2}$ as its "compactification radius". Postulating the effective (physically observable) metric as the one obtained by an average over $g_{00}$ involving many oscillation periods, the resulting space-time was found to inflate exponentially in $a_{1}$.

Considering the viability of such a scenario to describe the actual universe, a lot of questions and problems arise. Some of these have been posed (not answered) in Ref. 28]. Here, we would like to attack one such question, namely: how does non-gravitational matter "feel" the underlying signature oscillations? We will do a step towards an answer by choosing a minimally coupled scalar field $\phi$ with a self-interaction potential $V(\phi)$ as matter. This provides a first orientation, and we will leave the inclusion of higher spin fields (in particular the interesting case of fermions) to future work. Furthermore, we will consider the scalar field dynamics on a background metric of the type described above. This is easier than including the full back-reaction at the fundamental level, and will even help us exhibiting the structure that emerges. 
In Section 2, we will briefly review the scenario that has been worked out in Ref. [28]. Thereafter, in Section 3, we define what the dynamics of the scalar field at the fundamental level shall be, and perform a coarse graining type of average in order to obtain the effective field equation. The structure emerging is a standard scalar field equation, supplemented by a term $\tau \partial_{\tau} \phi$. In Section 4 , this correction is expressed in terms of a scalar $f$ which traces the hypersurfaces of signature change in the effective geometry. The result is a particular coupling of $\phi$ to the background scalar $f$. Formaly, $f$ turns out to satisfy the massive scalar field equation with $m_{f}^{2}=2 \Lambda / 11$, although it is not clear to what extent it develops features of a standard scalar field. Some of the structures appearing here are likely to carry over to less symmetric situations, as well as to models containing a richer matter sector. Finally, in Section 5 , we try to point out reasonable directions for future research.

\section{Review of signature-change-induced compacti- fication}

Let us collect some results from Ref. 28] that are essential for the purposes pursued here. As already mentioned in the introduction, we consider a background metric of the type

$$
d s_{\text {true }}^{2}=-s(\tau) d \tau^{2}+a_{1}(\tau)^{2} d \sigma_{3}^{2}+a_{2}(\tau)^{2} d \sigma_{6}^{2}
$$

where $d \sigma_{n}^{2}$ is the metric on the round unit n-sphere $\mathbf{S}^{n}$ (one may, for large $\tau$, ignore the curvature of $\mathbf{S}^{3}$ and thus approximate $d \sigma_{3}^{2}$ by a flat metric). As $\tau \rightarrow \infty$, the dominant behaviour is given by $a_{1} \rightarrow C \tau$ and $a_{2} \rightarrow(15 / \Lambda)^{1 / 2}$, the next order displaying damped oscillations such that $\delta a_{1} / a_{1} \sim \delta a_{2} / a_{2} \sim \Lambda^{2} \tau^{-2}$ during each period. These oscillations are induced by an infinite succession of signature changes such that $g_{00}^{\text {true }} \equiv-s= \pm 1$. Hence we have $s=1$ for the Lorentzian and $s=-1$ for the Euclidean periods. In the former case, $\tau$ is given by the cosmological proper time, in the latter case by its Euclidean analogue. The coordinate values at which a change of signature occurs are denoted by $\tau_{j}$, the corresponding interval sizes being $\Delta \tau_{j}=\tau_{j}-\tau_{j-1}(\mathrm{j}=1,2,3 \ldots)$.

For large $j$ (and hence large $\tau_{j}$ ) the mixed-signature Einstein field equations (with cosmological constant, but otherwise vaccum) imply to the lowest relevant order

$$
\Delta \tau_{j}=\sqrt{\frac{3}{2 \Lambda}}\left(\frac{1}{2 j}+(-)^{j} \frac{11}{12 j^{3 / 2}}\right),
$$


where Lorentzian periods correspond to even $j$, and Euclidean ones to odd $j$. There is a slight predominance of the Lorentzian over the Euclidean periods. Applying an appropriate average (coarse graining) to the metric (2.1) we found that a small part of $g_{00}$ survives and gives rise to the effective value

$$
g_{00}^{\mathrm{eff}}(\tau)=-<s(\tau)>=-\frac{11}{\Lambda \tau^{2}}
$$

The physical ten-metric resulting from this procedure is thus, for large $\tau$,

$$
d s_{\text {eff }}^{2}=-\frac{11}{\Lambda} \frac{d \tau^{2}}{\tau^{2}}+C^{2} \tau^{2} d \sigma_{3}^{2}+\frac{15}{\Lambda} d \sigma_{6}^{2} .
$$

After a transformation of the time coordinate

$$
\sqrt{\frac{11}{\Lambda}} \ln \left(\frac{\tau}{\tau_{0}}\right)=\eta
$$

it takes the form

$$
d s_{\mathrm{eff}}^{2}=-d \eta^{2}+\frac{K}{\Lambda} \exp \left(2 \sqrt{\frac{\Lambda}{11}} \eta\right) d \sigma_{3}^{2}+\frac{15}{\Lambda} d \sigma_{6}^{2}
$$

where $K$ can be given any value by a suitable choice of $\tau_{0}$. The physical four-metric is obtained by omitting the $d \sigma_{6}^{2}$-contribution in (2.4) or (2.6). It nicely displays inflationary expansion, although it must be said that a way out of this behaviour is not provided by the simple model we are considering. This and other problems have been discussed in Ref. [28]. The general scenario of compactification by signature change is likely to carry over to a large class of topologies, the major condition being that the internal space has non-zero Ricci curvature (and thus must be at least two-dimensional).

Let us at the end of this Section introduce the abbreviation

$$
f(\tau) \equiv<s(\tau)>=\frac{11}{\Lambda \tau^{2}}=\frac{11}{\Lambda \tau_{0}^{2}} \exp \left(-2 \sqrt{\frac{\Lambda}{11}} \eta\right)
$$

that will be used in what follows and note that, whenever we write $g_{00}^{\text {eff }}$ or $g_{\text {eff }}^{00}$, we refer to the metric in the form (2.4). 


\section{Scalar field effective dynamics}

Having a scheme at hand like the one described above, a natural question is whether one may recover physical laws that are compatible with our experience of space, time and matter interactions. The general advantages of the existence of compactified dimensions (a gauge theory of elementary particles arising at least partially from internal symmetries: see Ref. [27] for a recent review) are accompagnied here by the rather unusual idea that within each second of physical time (as measured by a clock) some underlying metric (namely (2.1)) undergoes a great number of oscillations between Lorentzian and Euclidean type, and only a small predominance of the former is responsible for the existence of a time evolution in the every-day sense. Even if the oscillations may not be observable (due to their extremely small time scales, and probably due to quantum effects as well [28]), one may ask how they affect the properties of matter, and whether they produce physical effects at the "effective" level. One such effect is the inflationary behaviour of (2.6) - at least in our simple model without any matter except a cosmological constant.

In order to proceed exploring the consequences of the scenario we described, let us study the behaviour of a minimally coupled real scalar field $\phi$. By this we mean that the metric (2.1) serves as a fixed background structure at the "true" level. The aim is to exhibit the dynamics of $\phi$ at the "effective" level (which is characterized by the metric (2.4), possibly without the $d \sigma_{6}^{2}$-term). The effective dynamics of $\phi$ would thus be accessible to physical predictions and measurements. Note that we neglect the back-reaction of the scalar field onto the metric. This approximation is good enough to represent a viable framework for our goals. The inclusion of $\phi$ into the full dynamics as well as the generalization to various types of interacting matter fields are not expected to provide drastic changes at the fundamental level at which the question is posed: what is the trace left by the signature oscillations in the observed world?

In order to be specific, let $(x, y)$ denote the coordinates on $\left(\mathbf{S}^{3}, \mathbf{S}^{6}\right), x^{0} \equiv \tau$, and let $\phi \equiv \phi(\tau, x, y)$. The effect of the existence of internal dimensions on observations in physical $(\tau, x)$-space is well known: Expanding $\phi$ in terms of appropriate modes on $\mathbf{S}^{6}$, one obtains an infinite "tower" of scalar fields corresponding to the structure of possible internal excitations (see e.g. Ref. [18]). This is logically independent of what we are aiming at, and may be performed at any stage of our considerations (preferably at the end). Hence, we will leave these issues aside, and simply allow $\phi$ to depend on all coordinates, as already indicated above. If one likes to study only the 
modes homogeneous on $\mathbf{S}^{6}$, one sets $\phi \equiv \phi(\tau, x)$. In even more drastic simplifications, one may let $\phi \equiv \phi(\tau)$, as is usually done in minisuperspace approaches to cosmology. The analysis given below will be uneffected by such choices.

The field equation at the "true" level shall be as simple and natural as possible. Hence, we assume the fundamental scalar field equation to be

$$
\square_{\text {true }} \phi \equiv g^{-1 / 2} \partial_{\mu} g^{1 / 2} g_{\text {true }}^{\mu \nu} \partial_{\nu} \phi=V^{\prime}(\phi),
$$

where $g \equiv\left|\operatorname{det}\left(g_{\mu \nu}^{\text {true }}\right)\right|$ and $V$ is a self-interaction potential. In the case of a free massive field, one would specify $V(\phi)=m^{2} \phi^{2} / 2$. This equation is meant to apply for the Lorentzian and the Euclidean domains separately. In other words, in (3.1) $g_{\mathrm{true}}^{00}=-s(\tau)$ is considered as a constant \pm 1 . Denoting the spatial part of the metric by $g_{i j}^{\text {true }}$, and pulling the constant $s$ out of the $\tau$-derivatives, we find

$$
g^{-1 / 2} \partial_{\tau} g^{1 / 2} \partial_{\tau} \phi=s\left(g^{-1 / 2} \partial_{i} g^{1 / 2} g_{\text {true }}^{i j} \partial_{j} \phi-V^{\prime}(\phi)\right) .
$$

This should be viewed as a second order time evolution equation. The coefficients - encoded in the $g_{\mu \nu}^{\text {true }}$ - perform continuous damped oscillations, except for $s(\tau)$ which is now allowed to undergo an infinite succession of jumps between 1 and -1 . The condition for the behaviour of the scalar field at the matching hypersurfaces $\tau=\tau_{j}$ shall be contituity of $\phi$ and existence of $\partial_{\tau} \phi$. As a consequence, $\partial_{\tau \tau} \phi$ becomes discontinuous, which is analogous to the evolution of the scale factors [28]. Note that, had we retained $g_{\text {true }}^{00}$ inside the derivatives in (3.1), an additional term containing $\delta\left(\tau-\tau_{j}\right)$ would have emerged and prevented the well-posed matching conditions associated with (3.2). This is in accordance with Refs. [29]- 30], where a purely time-dependent scalar is considered, and with Refs. [33]- 334, where the scalar field matching conditions are discussed in a two-dimensional model. In Ref. [33], by the way, it is shown that the jump conditions imply particle production when $\phi$ is a quantum field. (This is an aspect that seems worth being pursued further in the present context as well).

Having specified the "true" dynamics of $\phi$, we ask now for its behaviour if the observational resolution is such that $g_{00}^{\text {true }}$ is replaced by its average (2.3). Since we are interested in the limit of large $\tau$, the damped oscillations of the scale factors may be neglected. In Ref. [28], these oscillations have been studied in detail, and a straightforward application of the insights gained there reveals that $g^{-1 / 2} \partial_{\tau} g^{1 / 2}$ behaves as $3 / \tau+O\left(1 / \tau^{2}\right)$ just as if one had used $\hat{g}_{\text {eff }}^{-1 / 2} \partial_{\tau} \hat{g}_{\text {eff }}^{1 / 2}$ instead, where $\hat{g}_{\text {eff }}^{1 / 2} \equiv$ $g_{\mathrm{eff}}^{1 / 2}\left|g_{\mathrm{eff}}^{00}\right|^{1 / 2}$ (corresponding to the spatial part of (2.4). The only danger comes from $s$ 
at the right hand side of (3.2). Since the time scale (2.2) of the oscillations decreases to very small values, the observed (coarse grained) average of $\phi$ will experience some "inertia". Neglecting the small-scale wiggles in $\phi$, one may just replace $s$ by $\langle s\rangle$ from (2.3) in the version (3.2) of the field equation. However, this is $-g_{00}^{\text {eff }}$. Multiplying by $-g_{\mathrm{eff}}^{00}$, and reshuffling terms, the resulting effective scalar field equation is given by

$$
-g_{\mathrm{eff}}^{-1 / 2}\left|g_{\mathrm{eff}}^{00}\right|^{1 / 2} \partial_{\tau} g_{\mathrm{eff}}^{1 / 2}\left|g_{\mathrm{eff}}^{00}\right|^{1 / 2} \partial_{\tau} \phi+g_{\mathrm{eff}}^{-1 / 2} \partial_{i} g_{\mathrm{eff}}^{1 / 2} g_{\mathrm{eff}}^{i j} \partial_{j} \phi=V^{\prime}(\phi) .
$$

Thus, it becomes clear what has happened (to leading order in $\tau^{-1}$ ): The original $g_{\text {true }}^{00}$ has been replaced by $g_{\text {eff }}^{00}$, but such that part of it appears already inside the $\tau$-derivative. In order to restore a proper Laplacian (now with respect to the effective metric), a correction term $\left(\partial_{\tau}\left|g_{\text {eff }}^{00}\right|^{1 / 2}\right)\left|g_{\text {eff }}^{00}\right|^{1 / 2} \partial_{\tau} \phi \equiv(\Lambda / 11) \tau \partial_{\tau} \phi$ (using $g_{\mathrm{eff}}^{00}=-\Lambda \tau^{2} / 11$ ) is picked up. Thus (3.3) takes the form

$$
\left(\square_{\text {eff }}+\frac{\Lambda}{11} \tau \partial_{\tau}\right) \phi=V^{\prime}(\phi) .
$$

When expressed in terms of the effective cosmological proper time $\eta$ as appearing in (2.6), it may alternatively be written as

$$
\left(\square_{\text {eff }}+\sqrt{\frac{\Lambda}{11}} \partial_{\eta}\right) \phi=V^{\prime}(\phi) .
$$

Hence, the effective scalar field equation contains - in addition to the effective Laplacian - a first order time-derivative. Apart from this, the overall structure is obtained from the "true" equation (3.1) by simply replacing $\square_{\text {true }} \rightarrow \square_{\text {eff }}$.

The dynamical consequences of the additional term is estimated by noting that the pure time-derivative contribution of $\square_{\text {eff }}$ is

$$
-\frac{\Lambda}{11}\left(\tau^{2} \partial_{\tau \tau}+4 \tau \partial_{\tau}\right) \equiv-\left(\partial_{\eta \eta}+3 \sqrt{\frac{\Lambda}{11}} \partial_{\eta}\right) .
$$

The first derivatives act as damping terms (which prevent a purely time-dependent $\phi \equiv \phi(\tau)$ from rapidly "rolling down" the potential in most inflationary models; see e.g. Ref. [41]). Comparison with (3.4) or (3.5) shows that the correction terms increase these damping effects, i.e. ensure that $\phi$ varies a bit slower than it would without them. The actual large- $\tau$ behaviour of $\phi$ is not so important here, because one would anyway prefer to modify the model such that inflation comes to end. However, what is really interesting with (3.4) or (3.5) is its structure. This will be considered in the next Section. 


\section{The imprint of signature oscillations on matter}

The effective scalar field equation contains the derivative $\tau \partial_{\tau}$, hence a non-metric element. This is due to the fact that the hypersurfaces $\tau=$ const - although just a coordinate to label space-time points from an "effective level" geometrical point of view - are preferred: it is exactly at such hypersurfaces where the "microscopic" signature changes occur. Equation (3.4) indicates how matter "feels" these oscillations. Let us pose the question: What is the type of non-metric structure one expects to play some role in physical space-time? From the point of view we have adopted (in order to obtain effective physics by some coarse graining type of average), the only effective-level non-metric structures of interest are the location of the hypersurfaces where signature change occurs, and their "density" (which is represented by $g_{00}^{\text {eff }}$, i.e. by the quantity $f$ defined in (2.7)). Hence, we expect $f$ to play the role of a function on four- (or ten-) dimensional space-time that is produced by the signature oscillations and from which all their imprint on the effective physics may be derived. This argument is not restricted to the very simple FRW model we are considering. Hence, we expect (in less symmetric situations) a scalar function $f\left(x^{\mu}\right)$ to arise as a quantity encoding the location of the signature change hypersurfaces $(f=$ const $)$ as well as their density (presumably by its value). It is only the equation (2.7) that might be specific for our model.

Having identified $f$ as the "trace" of signature oscillations, it is easy to recast the effective scalar field equation (3.4) into a generally covariant form. Using $\partial_{\tau} f=$ $-22 /\left(\Lambda \tau^{3}\right)$ and $\partial^{\tau} f \equiv g_{\text {eff }}^{00} \partial_{\tau} f=2 / \tau$ (in general $\partial^{\mu} f \equiv g_{\text {eff }}^{\mu \nu} \partial_{\nu} f$ ), one finds

$$
\frac{\Lambda}{11} \tau \partial_{\tau}=\frac{\partial^{\mu} f}{2 f} \partial_{\mu}
$$

Hence, the final form of the scalar field equation is given by

$$
\left(\square_{\mathrm{eff}}+\frac{1}{2} f^{-1}\left(\partial^{\mu} f\right) \partial_{\mu}\right) \phi=V^{\prime}(\phi)
$$

It states that $\phi$ couples in this particular way to a background scalar field $f$. Since there is not much chance for additional non-metric structure to arise, more general types of matter fields are expected to pick up additional couplings of this type as well (at least to the lowest order in $\tau^{-1}$ ). Note that $f$ has an "absolute" (coordinate invariant) meaning, and hence may be called a scalar with respect to the effective geometry. In this sense, the effective physics is perfectly covariant under general coordinate transformations (as is (4.2)). 
In addition, $f$ carries dynamical information about the "true" background metric. This is just because it serves to describe the hypersurfaces of signature change which emerge from the (mixed-signature) Einstein field equations in a dynamical way. However, it is not clear whether there is a simple way to express this dynamical behaviour by means of differential equations or a Lagrangian formulation as a model for $\left(g_{\mu \nu}^{\text {eff }}, f\right)$ in less symmetric situations. Let us just mention three observations that might help exhibiting the nature of $f$.

Firstly, as far as I can see, (4.2) may not be obtained from a local Lagrangian by variation of $\phi$, the reason being that the operator $f^{-1}\left(\partial^{\mu} f\right) \partial_{\mu}$ is formally antihermitean. This may be contrasted to the case of a scalar in an external electromagnetic field where one encounters $i A^{\mu} \partial_{\mu}$, the $i$ assuring formal hermiticity and thus allowing $A^{\mu}$ to be coupled to a complex scalar.

As a second observation we remark that in the purely massive case $\left(V^{\prime}(\phi)=m^{2} \phi\right)$ the f-terms in the field equation (4.2) may be absorbed to lowest order into a rescaling of the field. Setting

$$
\phi=-\frac{1}{4} f \psi
$$

the field equation becomes

$$
\square_{\text {eff }} \psi=\left(m^{2}+O\left(\tau^{-2}\right)\right) \psi,
$$

without a $\partial_{\mu} \psi$ term. (This is somewhat analogous to the above-mentioned electromagnetic case where the identification $A_{\mu} \sim f^{-1} \partial_{\mu} f$ would render $A_{\mu}$ gauge trivial. Then (4.3) is the analogue of a local $\mathrm{U}(1)$ gauge transformation). Hence, one might try to consider rescaled quantities like $\psi$ (or even a conformally rotated metric) as the physical fields, rather than $\left(\phi, g_{\mu \nu}^{\mathrm{eff}}\right)$. To which extent this is possible and reasonable deserves further study.

The third observation concerns the status of $f$ "by itself" (i.e. without reference to $\phi)$. A simple calculation using (2.7) and (3.6) reveals that

$$
\square_{\mathrm{eff}} f=\frac{2 \Lambda}{11} f .
$$

Hence, at least in our simple model, $f$ satisfies the equation of a massive scalar field with $m_{f}^{2}=2 \Lambda / 11$. Due to the symmetries in the FRW-ansatz it is clear that only the homogeneous mode is excited $(f \equiv f(\tau))$. It would be interesting to know whether (4.5) carries over to less symmetric situations. (Recalling $f \equiv-g_{00}^{\text {eff }}, 4.5$ ) 
looks like a coordinate condition emerging from the proper-time nature of $\tau$ in the "true" geometry and some dynamical input). However, in any case such a dynamical equation would not apply without additional restrictions. Even in the FRW model, the general purely time-dependent solution to (4.5) is $f(\tau)=c_{1} \tau^{-1}+c_{2} \tau^{-2}$, whereas only its second part is realized.

To summarize, we have provided some evidence that the scenario of compactification by signature change leads to the appearance of an effective, dynamical ("signaton") scalar field $f$ that couples to matter. In the FRW model we used for all explicit computations, $f$ corresponds to the homogeneous mode of the (standard) massive scalar field equation with $m_{f}^{2}=2 \Lambda / 11$, although its dynamical nature in more general cases is unclear. In the concluding Section we will speculate on this last issue.

\section{Outlook}

We have already mentioned that we expect the basic structure emerging in our scenario to carry over to less symmetric situations. As in all inflationary models, the FRW geometry may serve to approximate physics locally. Globally, however, the geometry as well as the topology may be completely different (see e.g. Ref. 42]). In this sense, it is conceivable to encounter a global analogue $f\left(x^{\mu}\right)$ of the function $f(\tau)$ from (2.7), possibly subject to the massive scalar field equation (4.5) everywhere or in some large portion of total space-time. Such a "signaton" field would trace the global structure of the hypersurfaces of signature change.

It is thus of major importance for the scenario to find out which dynamical laws the effective structures are subject to, i.e. whether it is possible to formulate some effective dynamics for the system $\left(g_{\mu \nu}^{\mathrm{eff}}, f\right)$. One step towards this direction would be to find out whether $f$ is associated with some effective energy momentum tensor. In the FRW model, the energy momentum tensor of the four-dimensional part of the metric (2.4), is of the cosmological constant type $T_{\mu \nu}^{\mathrm{eff}}=\Lambda_{\text {eff }} g_{\mu \nu}^{\mathrm{eff}}$, with $\Lambda_{\text {eff }}=3 \Lambda / 11$. (Here we have neglected the curvature of the inflating $\mathbf{S}^{3}$, which is compatible with all the large- $\tau$ limits we have been considering in this paper). Hence, any attempt to interpret this as (partially) arising from $f$ would amount to set $T_{\mu \nu}(f) \sim g_{\mu \nu}^{\text {eff }}$. This is in turn not the standard scalar field energy momentur tensor. One possibility to 
represent it in terms of $f$ is an expression like $T_{\mu \nu}(f) \sim-g_{\mu \nu}^{\text {eff }}\left(\partial_{\rho} f\right)\left(\partial^{\rho} f\right) / f^{2}$, since

$$
f^{-2}\left(\partial_{\rho} f\right) \partial^{\rho} f=-\frac{4 \Lambda}{11}
$$

is a constant. Maybe in such an approach the field $f$ replaces the cosmological constant completely at the effective level. Another possibility would be to assign a potential $\sim \Lambda$ to $f$ and to note that the free part of the standard scalar field energy momentum tensor when written in terms of $f$ (namely $\left.-\left(\partial_{\mu} f\right)\left(\partial_{\nu} f\right)+g_{\mu \nu}^{\text {eff }}\left(\partial_{\rho} f\right)\left(\partial^{\rho} f\right) / 2\right)$ is of higher order $\left(\sim \tau^{-2}\right)$ than the potential contribution $(\sim \Lambda)$ and would not show up anyway in the large- $\tau$ approximation. If this idea goes through, $f$ would be treated as a scalar field with constant potential. However, as already stated in the preceeding Section, $f$ will in any case be forced to obey additional restrictions, and it may well turn out that it is in a rather strong way more a "function" of $g_{\mu \nu}^{\text {eff }}$ than a field degree of freedom by its own. In the FRW model, all these attempts coincide to leading order in $\tau^{-1}$, and one would have to study more general situations in order to distinguish between them.

A further obstruction against $f$ behaving like an ordinary scalar field is the fact that $f>0$. Furthermore, it is dimensionless and can be made a standard scalar only after a redefinition $f_{\text {scalar }}=\Lambda^{-1 / 4} f$. However, there is some freedom in trying whether a function $F(f)$ fits better into a convenient dynamical scheme. This may be seen by looking at the equations

$$
\square \ln f=\frac{6 \Lambda}{11}, \quad \square f^{3 / 2}=0 .
$$

Irrespective of these issues (that may contain aesthaetical as well as physical aspects), one would like to know whether $f$ or $F(f)$ develops some type of large-scale fluctuations or even wave-like modes.

As a last remark we add that it might be worth examining whether the appearance of an effective "signaton" field (giving rise to a preferred slicing!) has some implications on the problem of time (and related issues) when the "classical signature change model" is quantized [43]. Classical signature change occurs when the potential appearing in the Hamiltonian constraint (called $W$ in Ref. [28]) is zero. Quantization of this model amounts to replace $W \rightarrow|W|$ in the conventional Wheeler-DeWitt equation [40] (thus rendering the potential non-negative). Within this framework, one could try to extract something like $f$ on a full quantum or semi-classical level, and then re-examine all the conceptual questions encountered in quantum cosmology [44]- 46 . 


\section{References}

[1] E. Cremmer and B. Julia, "The SO(8) supergravity", Nucl. Phys. B 159, 141 (1979).

[2] A. Chodos and S. Detweiler, "Where has the fifth dimension gone?", Phys. Rev. D 21, 2167 (1980).

[3] P. G. O. Freund and M. A. Rubin, "Dynamics of dimensional reduction", Phys. Lett. 97 B, 233 (1980).

[4] P. G. O. Freund, "Kaluza-Klein Cosmologies", Nucl. Phys. B 209, 146 (1982).

[5] T. Appelquist and A. Chodos, "Quantum Effects in Kaluza-Klein Theories", Phys. Rev. Lett. 50, 141 (1983).

[6] T. Appelquist and A. Chodos, "Quantum dynamics of Kaluza-Klein theories", Phys. Rev. D 28, 772 (1983).

[7] T. Appelquist, A. Chodos and E. Myers, "Quantum instability of dimensional reduction", Phys. Lett. 127 B, 51 (1983).

[8] M. A. Rubin and B. D. Roth, "Fermions and stability in five-dimensional Kaluza-Klein theory", Phys. Lett. 127 B, 55 (1983).

[9] A. Chodos and E. Myers, "Gravitational Contribution to the Casimir Energy in Kaluza-Klein Theories", Ann. Phys. (N.Y.) 156, 412 (1984).

[10] A. Chodos and E. Myers, "Gravitational Casimir energy in non-Abelian KaluzaKlein theories", Phys. Rev. D 31, 3064 (1985).

[11] D. Sahdev, "Towards a realistic Kaluza-Klein cosmology", Phys. Lett. 137 B, 155 (1984).

[12] R. B. Abbott, S. M. Barr and S. D. Ellis, "Kaluza-Klein cosmologies and inflation", Phys. Rev. D 30, 720 (1984).

[13] E. W. Kolb, D. Lindley and D. Seckel, "More dimensions - Less entropy", Phys. Rev. D 30, 1205 (1984).

[14] D. Sahdev, "Perfect fluid higher-dimensional cosmologies", Phys. Rev. D 30, 2495 (1984). 
[15] M. Yoshimura, "Effective action and cosmological evolution of scale factors in higher-dimensional curved space", Phys. Rev. D 30, 344 (1984).

[16] R. A. Matzner and A. Mezzacappa, "Professor Wheeler and the Crack of Doom: Closed Cosmologies in the 5-d Kaluza-Klein Theory", Found. Phys. 16, 227 (1986).

[17] K. Maeda, "Stability and attractor in a higher-dimensional cosmology:I,II", Class. Quantum Grav. 3, 233 (1986); Class. Quantum Grav. 3, 651 (1986).

[18] D. Bailin and A. Love, "Kaluza-Klein theories", Rep. Prog. Phys. 50, 1087 (1987).

[19] D. L. Wiltshire, "Global properties of Kaluza-Klein cosmologies", Phys. Rev. D 36, 1634 (1987).

[20] M. Szydlowski and M. Biesiada, "Inflation as a dynamical effect of higher dimensions", Phys. Rev. D 41, 2487 (1990).

[21] O. Bertolami, Yu. A. Kubyshin and J. M. Mourão, "Stability of compactification in Einstein-Yang-Mills theories after inflation", Phys. Rev. D 45, 3405 (1992).

[22] O. Bertolami, J. M. Mourão and Yu. A. Kubyshin, "On the stability of compactification after inflation", in: H. Sato and T. Nakamura (eds.), Proceedings of the 6th Marcel Grossmann Meeting 1991, World Scientific (Singapore, 1992), p. 625 .

[23] Q. Shafi and C. Wetterich, "Cosmology from higher-dimensional gravity" Phys. Lett. 129 B, 387 (1983).

[24] Q. Shafi and C. Wetterich, "Inflation with higher dimensional gravity", Phys. Lett. 152, 51 (1985).

[25] M.Reuter and C. Wetterich, "Classical stability for spontaneous compactification in higher derivative gravity", Nucl. Phys. B 289, 757 (1987).

[26] Q. Shafi and C. Wetterich, "Inflation from higher dimensions", Nucl. Phys. B 289, 787 (1987). 
[27] M. J. Duff, "Kaluza-Klein theory in perspective", Talk delivered at the "Oskar Klein Centenary Nobel Symposium", Stockholm, September 1994, preprint hepth/9410046.

[28] F. Embacher, "Signature change induces compactification", University Vienna preprint UWThPh-1994-47 (also available as gr-qc/9410012).

[29] G. Ellis, A. Sumeruk, D. Coule and C. Hellaby, "Change of signature in classical relativity", Class. Quantum Grav. 9, 1535 (1992).

[30] G. F. R. Ellis, "Covariant Change of Signature in Classical Relativity", Gen. Relativ. Gravit. 24, 1047 (1992).

[31] T. Dereli and R. W. Tucker, "Signature dynamics in general relativity", Class. Quantum Grav. 10, 365 (1993).

[32] R. Kerner and J. Martin, "Change of signature and topology in a fivedimensional cosmological model", Class. Quantum Grav. 10, 2111 (1993).

[33] T. Dray, C. A. Manogue and R. W. Tucker, "Particle Production from Signature Change", Gen. Relativ. Gravit. 23, 967 (1991).

[34] T. Dray, C. A. Manogue and R. W. Tucker, "Scalar field equation in the presence of signature change", Phys. Rev. D 48, 2587 (1993).

[35] C. Hellaby and T. Dray, "Failure of standard conservation laws at a classical change of signature", Phys. Rev. D 49, 5096 (1994).

[36] T. Dray and C. Hellaby, "The patchwork divergence theorem", to appear in $J$. Math. Phys, preprint gr-qc/9404002.

[37] G. W. Gibbons and J. B. Hartle, "Real tunneling geometries and the large-scale topology of the universe", Phys. Rev. D 42, 2458 (1990).

[38] S. A. Hayward, "Signature change in general relativity", Class. Quantum Grav. 9, 1851 (1992).

[39] S. A. Hayward, "On cosmological isotropy, quantum cosmology and the Weyl curvature hypothesis", Class. Quantum Grav. 10, L7 (1993). 
[40] F. Embacher, "Comments on the multi-dimensional Wheeler-DeWitt equation", Talk given at the International School-Seminar "Multidimensional Gravity and Cosmology", Yaroslavl, June 1994, to appear in the proceedings, preprint gr-qc/9409016.

[41] R. H. Brandenberger, "Inflation and cosmic strings: Two mechanisms for producing structure in the universe", Int. J. Mod. Phys. A 2, 77 (1987).

[42] A. Linde, "Inflation and quantum cosmology", Physica Scripta T 36, 30 (1991).

[43] J. Martin, "Hamiltonian quantization of general relativity with the change of signature", Phys. Rev. D 49, 5086 (1994).

[44] C. J. Isham, "Canonical quantum gravity and the problem of time", in: L. A. Ibort and M. A. Rodriguez (eds.), Integrable systems, quantum groups, and quantum field theories, Kluwer Academic Publishers (London, 1993), 157-287.

[45] K. V. Kuchar, "Time and interpretations of quantum gravity", in: G. Kunstatter et. al. (eds.), Proceedings of the 4 th Canadian conference of general relativity and relativistic astrophysics, World Scientific (Singapore, 1992), 211 - 314.

[46] A. Ashtekar and J. Stachel (eds.), Conceptual Problems of Quantum Gravity, Birkhäuser (Boston, 1991). 